People living with a lung condition who attended any type of support group had significantly greater quality of life at 6 months compared to control.

Those attending groups maintained quality of life throughout the study whereas quality of life decreased by more than $20 \%$ for patients in control group.

Those attending standard groups maintained self-efficacy whereas there was a decrease of $17 \%$ for those in control group.

For each pound invested in the integrated support groups there is a return of a minimum $£ 3.43$ and a maximum of $£ 9.36$.

For each pound invested in the integrated groups, there is a net gain of $£ 8.01$ in social return.

Lessons learnt Integrated respiratory patient support groups is a cost effective programme which has positive outcomes in terms of self-efficacy, health outcomes and wellbeing for attendees, providing cost savings and wider social benefits to local communities.

\section{P202 OPTIMISING SERVICE DELIVERY IN ASTHMA AND COPD CONSENSUS-DRIVEN RECOMMENDATIONS FOR FUTURE SERVICE DEVELOPMENT}

${ }^{1} \mathrm{M}$ Ledson, ${ }^{2} \mathrm{~L}$ Baskaran, ${ }^{3} \mathrm{C}$ Dunford, ${ }^{4} \mathrm{~S}$ Gwynn, 5 Khambh , ${ }^{5} \mathrm{~S}$ Prigmore, ${ }^{7} \mathrm{~S}$ Scullion. ${ }^{1}$ Liverpool Heart and Chest Hospital, Liverpool, UK; ${ }^{2}$ The James O'riordan Medical Centre, Sutton, UK; ${ }^{3}$ The Viceroy Surgery, Birmingham, UK; ${ }^{4}$ Triducive Limited, St Albans, UK; ${ }^{5}$ NHS London Procurement Partnership, London, UK; ${ }^{6}$ St Georges Hospital, Tooting, UK; ${ }^{7}$ Glenfield Hospital, Leicester, UK

\subsection{6/thoraxjnl-2016-209333.345}

Introduction and objectives Asthma and COPD present a significant resource impact to the NHS. Earlier diagnosis may reduce morbidity and improve quality of life. In the UK, premature mortality from COPD is almost twice the European average and for asthma over 1.5 times the European average. This project sponsored by Teva Respiratory aims to identify differences in perceptions of various stakeholder groups regarding effective outcome improvements in asthma and COPD and make relevant recommendations.

Methods This group met with the objective of defining consensus statements for the future development of services in asthma and COPD. These statements were tested across a broad respondent sample by questionnaire. A Delphi methodology was used to assess levels of agreement with each statement. Questionnaires were offered to health care professionals across specialties reflecting the roles of this group for completion as paper documents at Teva Respiratory sponsored UK meetings between June 2015 and January 2016.

Results 184 respondents, split across varied professional roles, completed questionnaires. 24 out of 42 statement scores (57\%) exceeded the $66 \%$ agreement threshold and are thus regarded as supportive of the statements. Some variance was seen in responses between care settings (Figure 1), with primary care respondents commonly indicating lower levels of agreement than their secondary care colleagues (24 out of 42 statements $(57 \%)$ ).

Conclusions Most respondents indicate that it is possible to deliver effective care across all care settings that the patient will encounter. The need for further development of local integrated care approaches is well recognised. Respondents are ambivalent regarding the prioritisation of asthma and COPD, the variance may reflect differences in prioritisation between localities. There is strong agreement that definition of appropriate outcomes will support value-based care models and that interaction between professions is critical to effective integration of care. Respondents agreed there is a sound rationale for the use of branded inhaler therapies in asthma and COPD, which may liberate finite resources for other areas.

Based on this consensus exercise, 10 key recommendations for optimising outcomes in asthma and COPD are offered.

\section{P203 ACUTE ONCOLOGY SERVICES AND THE CHEST PHYSICIAN}

JA Benjamin, K Wingfield, C Garman. Cwm Taf University Health Board, Pontyclun, UK

\subsection{6/thoraxjnl-2016-209333.346}

Background At our trust (Popn 289400, x2 district general hospitals,) the Acute Oncology Service (AOS) began formally in June 2015. The team consists of 6 members, 3 consultants $(1$ session per week each ; $\mathrm{x} 1$ clinical lead at both sites and one clinical lead for metastatic spinal cord compression across both sites,) 2 clinical nurse specialists full time, 1 data coordinator full time. One of the 3 consultants is a chest physician (author.)

- In September 2014 a formal expression of need for AOS development was accepted and supported by Macmillan for a 3 year fixed term project.

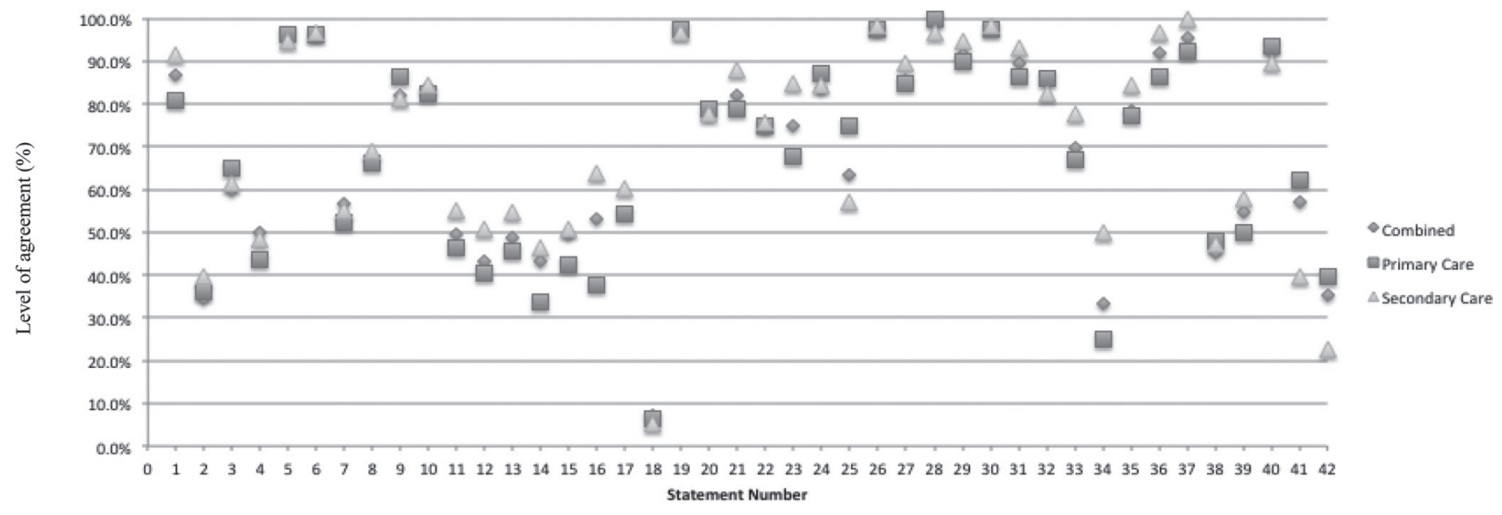

Abstract P202 Figure 1 Care settings comparision 
- There was a well supported process of induction for the nurses and introduction of the service to the Health Board prior to becoming clinically available in September 2016.

\section{Outcomes}

- 569 patients have been seen since clinically active (Sept 2015)

- Median reduction in length of hospital stay (LOS) from 11 to 5 days for patients with carcinoma of unknown primary (CUP)

- Median LOS since introduction of service is 5 days for all cancer diagnoses. This equates to a 1 day reduction in LOS. Median LOS in preceding years 2011-2015 = 6 days

- The largest number of refrrals to the service has been for patients with lung cancer (21\%) - see Table

Conclusions/personnel reflections An effective AOS service improves quantatative outcomes(reduced LOS, efficient processing of CUP patients,) and enhances qualititative outcomes for patients (advocates for CUP patients, better communication*)

The majority of cancers dealt with by the AOS service are lung cancer

The outcomes above are almost exclusivley down to the AOS nurses but of all medical and surgical specialities, chest physicians (who deal with lung cancer) are ideally placed as clinical leads for this service due to their cancer experience and established links with radiology,pathology and palliative care (Author's own opinion.)

For present and future AOS services, this team would reccommend that an amenable/enthusisastic chest physician would be a valuable asset to the service

* Patient feedback can be provided on request
P204

\section{QUALITY IMPROVEMENT PROJECT FOR EMERGENCY} OXYGEN DELIVERY ON A RESPIRATORY WARD

KE Hutchinson, S Craik, K Srinivasan, H Moudgil, N Ahmad. Princess Royal Hospital, Telford, UK

10.1136/thoraxjnl-2016-209333.347

Background British Thoracic Society (BTS) guidelines state that oxygen should be used to treat hypoxaemia and prescribed to a target saturation range. ${ }^{1}$ Patients at risk of type 2 respiratory failure should target $88-92 \%$, with the rest $94-98 \%$. In the BTS national audit in 2013, out of 6214 patients, 55\% had oxygen prescribed and $52 \%$ were prescribed and delivered to within a target saturation range. ${ }^{2}$

Methods We ran a Quality Improvement Project (QIP) involving three PDSA cycles to improve the delivery of oxygen to patients on the Respiratory Ward at the Princess Royal Hospital, Telford.

We set our standards as:

1. $90 \%$ of patients receiving oxygen have it prescribed on a drug chart

2. $100 \%$ of patients prescribed oxygen have a documented target saturation range

3. $100 \%$ of patients have oxygen delivered appropriately to target

The QIP process commenced in Autumn 2015. After the first cycle we used bedside prompt cards and delivered teaching sessions with doctors, nurses and healthcare assistants (HCAs). After the second cycle we appointed a nurse, HCA and two FY1 Abstract P203 Table 1 Distribution of patients referred to our AOS by their major cancer site
Major Cancer Site (Drill Down Age-Range)

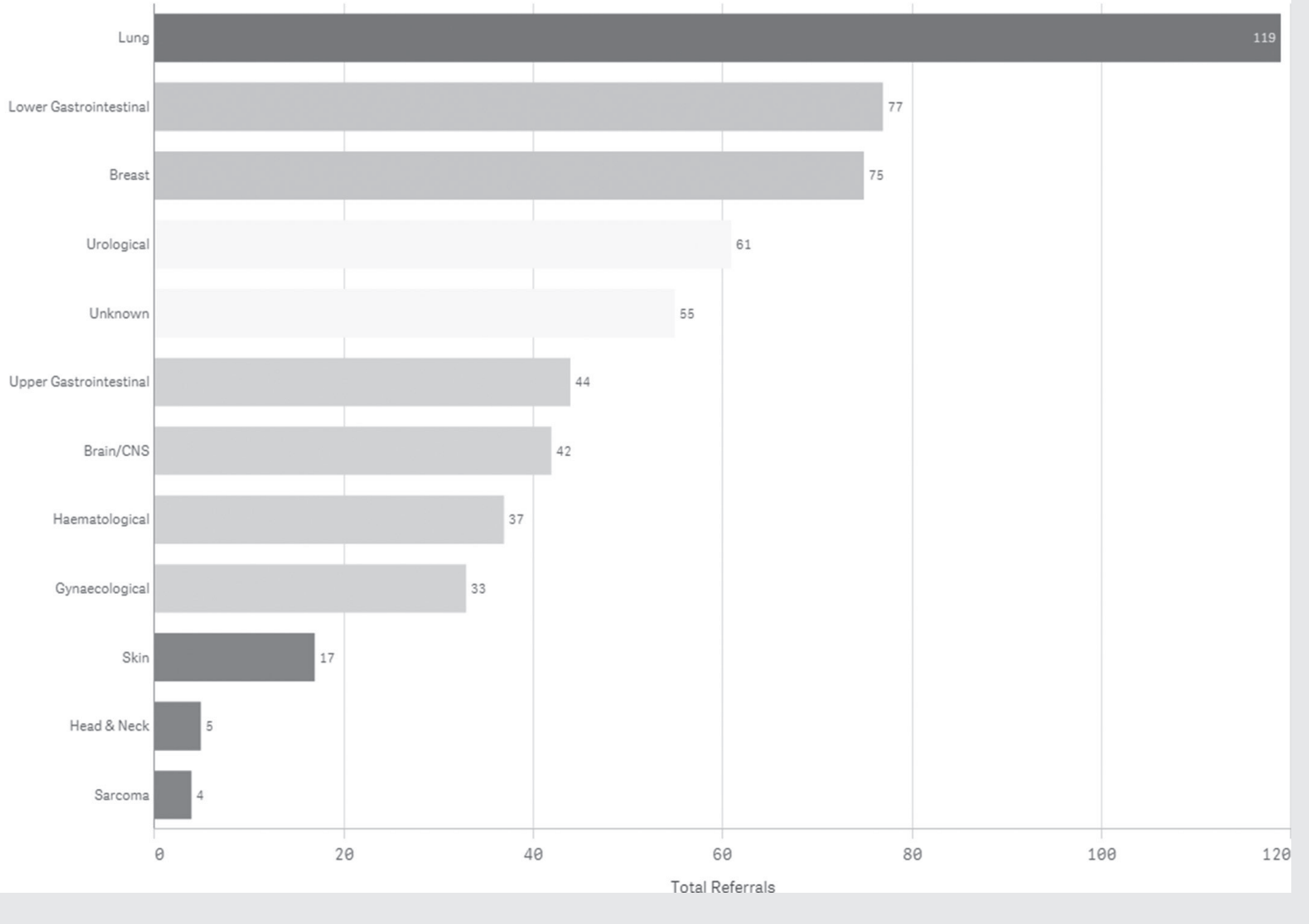

